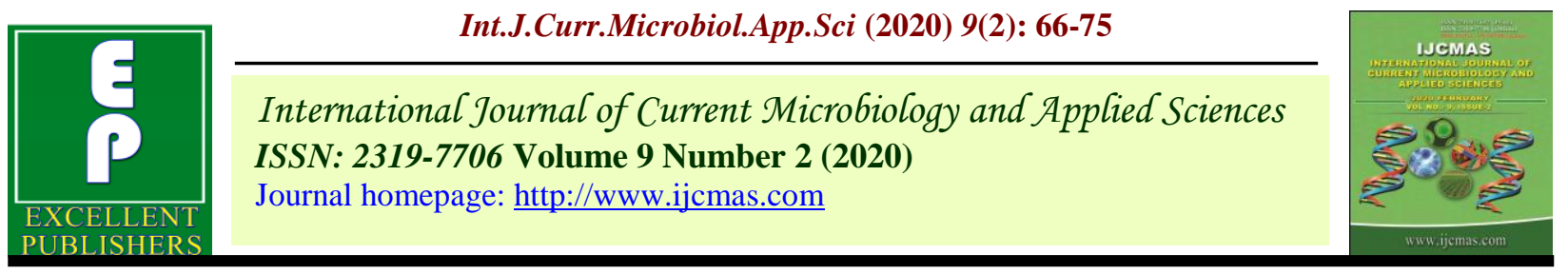

Original Research Article

https://doi.org/10.20546/ijcmas.2020.902.009

\title{
Correlation and Path Coefficient Analysis for Seed Yield and Fibre Traits in Linseed (Linum usitatissimum L.)
}

\author{
Navdeep Kaur* and Satish Paul \\ Department of Crop Improvement, CSK Himachal Pradesh Krishi Vishvavidyalaya, \\ Palampur-176062, India \\ *Corresponding author
}

\section{A B S T R A C T}

\section{Keywords}

Genotypic correlation coefficient,

Phenotypic

correlation, Harvest index, Aerial biomass

Article Info

Accepted:

05 January 2020

Available Online:

10 February 2020
30 genotypes were evaluated to understand the nature and magnitude of association for seed yield and fibre traits in linseed (Linum usitatissimum L). Genotypic and phenotypic correlation coefficients obtained between different traits was similar in direction, while in magnitude, genotypic correlation higher than the corresponding phenotypic correlations. Seed yield per plant showed positive and significant correlation with harvest index, 1000-seed weight, straw yield, aerial biomass, seeds per capsule, capsules per plant, days to 50 per cent flowering and days to 75 per cent maturity while, plant height showed significant negative correlation. On the basis of path coefficient analysis, harvest index followed by aerial biomass exhibited the highest direct effects on seed yield per plant.

\section{Introduction}

Linseed (Linum usitatissimum L.) commonly known as Alsi or Flax, is a multi-purpose rabi oilseed crop, which belongs to the family Linaceae having 14 genera (Gill 1987). Linum has over 200 species with Linum angustifolium Huds $(\mathrm{n}=15)$ being its probable progenitor, native to Mediterranean region and Southwest Asia. Linum usitatissimum is the only economically significant species of the family cultivated for oil and fibre. Linseed contains about 36 to $48 \%$ oil content which is high in unsaturated fatty acids, especially linolenic acid (Khan et al., 2010). It has drying and hardening properties which is emanated from its high linolenic acid content, thus is mostly used for industrial purposes such as manufacturing of paints, varnishes, soaps and printing ink. Every part of the linseed plant is utilized commercially either directly or after processing. The fibre extracted from the stem is lustrous and blends very well with wool, silk, cotton etc. Strong twines, canvas, suiting, shirting and various products used for defence purpose are manufactured from linseed fibre. The byproduct, oil cake is a valuable dairy feed 
containing 36 per cent protein, of which 85 per cent is digestible.

The current world wide acreage is only about 2.63 million ha, with total global production of 2.70 million tonnes. India holds fifth rank in area with 285.50 thousand ha $(10.80 \%)$ after Canada (23.64\%), Kazakhstan (21.20\%), Russia (16.81\%) and China (11.80\%) whereas in terms of productivity India $(541.0 \mathrm{~kg} / \mathrm{ha})$ is far behind to Switzerland, United Kingdom, Egypt, Canada, China and Russia (FAO Stat 2018). The area under linseed cultivation in Himachal Pradesh is 0.70 thousand ha and production is 200 tonnes with an average productivity of $243.0 \mathrm{~kg} / \mathrm{ha}$.

In Himachal Pradesh, its cultivation is mainly concentrated in the Palampur area of Kangra and Mandi district. However, its average productivity in India as well as in Himachal Pradesh is very low, because of various factors such as narrow genetic base, raising of crop by the resource poor farmers in marginal and sub-marginal areas, non-availability of improved varieties having resistance to biotic and abiotic stresses, non-adoption of plant protection measures and improved agrotechniques etc.

To overcome the poor yield levels, development of high yielding varieties becomes the top priority. Selection for yield via. highly correlated characters becomes easy if the contribution of different characters to yield is quantified using path coefficient analysis (Dewey and Lu 1959). In fact, practically selection criteria will contribute to selection based on direct effects (Yucel 2004; Copur and Oglakci 1998). However, simple correlation coefficients and analysis of variance may not evolve satisfactory findings in uncovering the real interrelationships among the various traits. So path coefficient analysis technique is a statistical approach which is based on multiple regression and is useful for revealing the direct and indirect effects of the variables in a network of factors like agro/morpho/physio/biochemical traits which is able to separate correlation coefficients into their components of direct and indirect effects (Dewey and Lu 1959; Wright 1960).

Keeping this in view, the experiment was conducted to

(a) understand the nature and magnitude of association for yield and fibre traits in linseed, and

(b) identify promising genotypes for direct or indirect use in linseed breeding programme.

\section{Materials and Methods}

Study material comprised of 30 linseed (Linum usitatissimum L.) genotypes, amongst which Nagarkot and JRF-4 were used as check. The investigation was carried out at the Experimental Farm of the Department of Crop Improvement, CSK HPKV, Palampur during rabi season 2015-16. The experiment was laid out in randomized block design with three replications. Each genotype was sown in four rows with the plot size of $1 \times 1 \mathrm{~m}^{2}$ with row to row and plant to plant spacing of $25 \mathrm{~cm}$ and $5 \mathrm{~cm}$ respectively.

Observations were recorded for different quantitative characters on five randomly selected plants for all the genotypes except for days to 50 per cent flowering, days to 75 per cent maturity, 1000-seed weight and oil content (\%) which was recorded on plot basis. The parameters taken at plant basis are primary branches per plant, secondary branches per plant, plant height $(\mathrm{cm})$, technical height $(\mathrm{cm})$, capsules per plant, seeds per capsule, straw yield (g), seed yield per plant $(\mathrm{g})$, retted straw yield (g), fibre yield $(\mathrm{g})$, aerial biomass $(\mathrm{g})$, harvest index $(\%)$. 


\section{Statistical analysis}

Phenotypic and genotypic coefficients of correlation were worked out by following the procedure of Al- Jibouri et al., (1958) and Dewey and Lu (1959). Path coefficient is a standardized partial regression coefficient and as such it is a measure of direct and indirect effects of a set of variables (component characters) on a dependent variable such as grain yield. Direct and indirect effects of component characters on grain yield were computed using appropriate correlation coefficient of different component characters as suggested by Wright (1921) and elaborated by Dewey and $\mathrm{Lu}$ (1959). The statistical analysis was performed by statistical software WINDOWSTAT 8.0 version.

\section{Results and Discussion}

The results on correlations at phenotypic and genotypic levels computed for all possible paired combinations are presented in Table 2. Genotypic correlation coefficients in general were higher than the phenotypic correlation which revealed that though there is a strong inherent association between various characters, the phenotypic expression of the correlation modified under the influence of environment. Similar findings were reported by Sohan et al., (2004), Joshi (2004) and Kumar and Paul (2016). The effective yield improvement would be achieved through the characters which have significant and positive correlation with yield and other economic traits.

At phenotypic level, seed yield per plant showed significant positive correlations with capsules per plant, seeds per capsule, 1000seed weight, aerial biomass, harvest index, days to 50 per cent flowering, days to 75 per cent maturity and straw yield, whereas seed yield per plant showed significant negative correlation with plant height. At genotypic level, the estimates of correlation coefficients were generally similar to those observed at phenotypic level. The significant positive correlations among different characters have been reported by different workers. Akbar et al., (2003) reported that seed yield per plant was positively associated with number of seeds per capsule and number of capsules per plant. Kant et al., (2008) reported significant and positive correlation of seed yield with number of capsules per plant, number of seeds per capsule, aerial biomass, harvest index, number of primary branches per plant and test weight. Significant positive correlations of seed yield with number of capsules per plant was reported by many workers such as, Mahto and Rahaman (1998), Gupta et al., (1999), Naik and Satapathy (2002), Sarkar (2005), Rao (2007), Gauraha and Rao (2011), Pali and Meheta (2013), Belete and Yohannes (2013), Rajanna et al., 2014 and Naik et al., (2016), with 1000-seed weight as reported by Badwal et al., (1970), Chandra (1978), Mahto and Rahaman (1998), Naik and Satapathy (2002), Gauraha and Rao (2011) and Pali and Meheta (2013) which is in confirmation with the present findings. Sarkar (2005), Gauraha and Rao (2011), Paul et al., (2015) and Paul et al., (2017) reported that plant height was positively correlated with seed yield which is not in confirmation with the results of present study, whereas Kumar and Paul (2016) reported negative significant correlation of plant height with seed yield per plant which is in due confirmation with the results of present study,

At phenotypic level, significant positive correlation was observed for days to 50 per cent flowering with days to 75 per cent maturity, primary branches per plant, secondary branches per plant, aerial biomass, straw yield, 1000-seed weight and retted straw yield; days to 75 per cent maturity with primary branches per plant, aerial biomass, straw yield, 1000-seed weight and retted 
straw yield; plant height with technical height, straw yield and retted straw yield; technical height with straw yield and retted straw yield; primary branches per plant with 1000 -seed weight; capsules per plant with seeds per plant and aerial biomass; seeds per capsule with harvest index; aerial biomass with straw yield, fibre yield and retted straw yield; straw yield with fibre yield and retted straw yield; 1000-seed weight with harvest index, and fibre yield with retted straw yield.

Similarly, significant negative correlation was observed for days to 50 per cent flowering with oil content and fibre yield; days to 75 per cent maturity with oil content; plant height with seeds per capsule and harvest index; technical height with seeds per capsule and harvest index; primary branches per plant with oil content; straw yield with harvest index, and harvest index with retted straw yield.

At genotypic level, significant positive correlation was observed for days to 50 per cent flowering with days to 75 per cent maturity, primary branches per plant, secondary branches per plant, aerial biomass, straw yield, harvest index, 1000-seed weight and retted straw yield; days to 75 per cent maturity with primary branches per plant, aerial biomass, straw yield, 1000-seed weight and retted straw yield; plant height with technical height, straw yield and retted straw yield, technical height with straw yield and retted straw weight; primary branches per plant with 1000-seed weight and retted straw yield; capsules per plant with seeds per plant, aerial biomass, straw yield and retted straw yield; seeds per capsule with harvest index and oil content; aerial biomass with straw yield, fibre yield and retted straw yield; straw yield with fibre yield and retted straw yield; 1000-seed weight with harvest index, and fibre yield with retted straw yield.
Significant positive correlation was observed for days to 50 per cent flowering with days to 75 per cent maturity was reported by Savita (2006), Gauraha and Rao (2011), Bibi et al., (2013) and Gul et al., (2016) which is in due confirmation with the results of present study. Significant positive correlation for days to 75 per cent maturity with primary branches per plant was observed by Gauraha and Rao (2011) which is in confirmation with the results of present study. Gauraha and Rao (2011) and Pali and Meheta (2013) observed that number of primary branches per plant had positive significant correlation with number of capsules per plant, which contradicts present findings. Akbar et al., (2001) also observed seed yield per plant was positively associated with number of seeds per pod, number of pods per plant and number of branches per plant. Similarly, Paul et al., (2017) reported significant positive correlations of capsules per plant with seeds per capsule, whereas Kumar and Paul (2016) and Bindra and Paul (2017) reported significant negative correlations which is not in confirmation with the results of present study.

The direct and indirect effects of genotypic path coefficient were higher in magnitude than the corresponding phenotypic path coefficients (Table 2). Similar finding with respect to path coefficients have also been reported by Gauraha and Rao (2011) and Reddy et al., (2013). In the present study, residual effects for path coefficients was low (0.029) indicating that almost all the contributing traits have been covered for explaining their contribution to the seed yield. At phenotypic level, harvest index followed by aerial biomass, oil-content, days to 50 per cent maturity, seeds per capsule and plant height, while days to 50 per cent flowering had the highest negative direct effect on seed yield. This indicated significant role of accumulation of harvest index and its partitioning into seed yield. 
Table.1 List of 30 germplasm accessions

\begin{tabular}{|c|c|c|}
\hline S.No. & Genotypes & Parentage/Source \\
\hline 1 & KL-274 & Giza- 6 × Giza-7 \\
\hline 2 & KL-275 & Giza- 6 × Belinka-60 \\
\hline 3 & KL-276 & Natasja $\times$ Faiking \\
\hline 4 & KL-277 & Giza-5 × Flak-1 \\
\hline 5 & KL-278 & Giza- $5 \times$ Aoyogi \\
\hline 6 & KL-279 & Mariena $\times$ Giza-5 \\
\hline 7 & KL-280 & Giza-7 × Belinka \\
\hline 8 & KL-281 & Giza-5 $\times$ Faiking \\
\hline 9 & KL-282 & Giza- $8 \times$ Aoyogi \\
\hline 10 & KL-283 & Giza- $8 \times$ Faiking \\
\hline 11 & KL-284 & Rajeena $\times$ Him Alsi- 2 \\
\hline 12 & KL-285 & Binwa $\times$ Him Alsi-2 \\
\hline 13 & KL-288 & $(\mathrm{TL}-43 \times$ Binwa $) \times \mathrm{TL}-43$ \\
\hline 14 & KL-302 & $($ Surbhi $\times$ LC-2232 $) \times$ Ariane \\
\hline 15 & KL-303 & Hermis $\times$ Surbhi \\
\hline 16 & JRF-1 & CRIJAF, Barrackpore (WB) \\
\hline 17 & JRF-2 & CRIJAF, Barrackpore (WB) \\
\hline 18 & JRF-3 & CRIJAF, Barrackpore (WB) \\
\hline 19 & SLS-V & Sagar, JNKVV (MP) \\
\hline 20 & K-1(Raja) & Exotic flax material \\
\hline 21 & Ottawa 77013 & Exotic flax material \\
\hline 22 & $\mathrm{~B}^{14}$ Burke & Exotic flax material \\
\hline 23 & Stewart & Exotic flax material \\
\hline 24 & Pale Blue & Exotic flax material \\
\hline 25 & Kenya & Exotic flax material \\
\hline 26 & Wilden & Exotic flax material \\
\hline 27 & Birio & Exotic flax material \\
\hline 28 & Barnes & Exotic flax material \\
\hline 29 & Nagarkot* & New River $\times$ LC-216 \\
\hline 30 & JRF-4* & CRIJAF, Barrackpore (WB) \\
\hline
\end{tabular}


Table.1 Estimates of correlation coefficients at phenotypic (P) and genotypic (G) levels among different traits of linseed

\begin{tabular}{|c|c|c|c|c|c|c|c|c|c|c|c|c|c|c|c|c|}
\hline Characters & & $\begin{array}{c}\text { Days to } \\
75 \text { per } \\
\text { cent } \\
\text { maturity }\end{array}$ & $\begin{array}{c}\text { Plant } \\
\text { height } \\
(\mathrm{cm})\end{array}$ & $\begin{array}{c}\text { Technical } \\
\text { height } \\
\text { (cm) }\end{array}$ & $\begin{array}{c}\text { Primary } \\
\text { branches } \\
\text { per plant }\end{array}$ & $\begin{array}{c}\text { Secondary } \\
\text { branches } \\
\text { per plant }\end{array}$ & $\begin{array}{c}\text { Capsules } \\
\text { per } \\
\text { plant }\end{array}$ & $\begin{array}{c}\text { Seeds } \\
\text { per } \\
\text { capsule }\end{array}$ & $\begin{array}{c}\text { Aerial } \\
\text { biomass } \\
\text { (g) }\end{array}$ & $\begin{array}{c}\text { Straw } \\
\text { yield } \\
(\mathrm{g})\end{array}$ & $\begin{array}{c}\begin{array}{c}\text { Oil } \\
\text { content } \\
(\%)\end{array}\end{array}$ & $\begin{array}{c}1000 \\
\text { seed } \\
\text { weight } \\
(\mathrm{g})\end{array}$ & $\begin{array}{c}\text { Fibre } \\
\text { yield } \\
\text { (g) }\end{array}$ & $\begin{array}{c}\text { Harvest } \\
\text { index } \\
(\%)\end{array}$ & $\begin{array}{c}\text { Retted } \\
\text { straw } \\
\text { yield } \\
(\mathrm{g})\end{array}$ & $\begin{array}{c}\text { Correlation } \\
\text { with } \\
\text { seed } \\
\text { yield } \\
\text { per plant }\end{array}$ \\
\hline \multirow{2}{*}{$\begin{array}{l}\text { Days to } \\
50 \% \\
\text { flowering }\end{array}$} & $\mathbf{P}$ & $0.836^{* *}$ & -0.04 & 0.072 & $0.559^{* *}$ & $0.246^{*}$ & 0.045 & -0.087 & $0.339^{* *}$ & $0.214^{*}$ & $-0.441^{* *}$ & $0.344^{* *}$ & $-0.224^{*}$ & 0.199 & $0.331^{* *}$ & $0.299^{* *}$ \\
\hline & G & $0.889^{\text {** }}$ & -0.024 & 0.095 & $0.607^{* *}$ & $0.294^{* *}$ & 0.039 & -0.121 & $0.386^{* *}$ & $0.238^{*}$ & $-0.480^{* *}$ & $0.361^{* * *}$ & $-0.243^{*}$ & $0.223^{*}$ & $0.389^{* * *}$ & $0.331^{* *}$ \\
\hline \multirow{2}{*}{$\begin{array}{l}\text { Days to } \\
\text { maturity }\end{array}$} & $\mathbf{P}$ & & -0.017 & 0.065 & $0.669^{* * *}$ & 0.036 & 0.146 & -0.029 & $0.473^{* *}$ & $0.309^{* *}$ & $-0.384^{* *}$ & $0.294^{* *}$ & -0.111 & 0.158 & $0.491^{* *}$ & $0.359^{* * *}$ \\
\hline & G & & -0.014 & 0.079 & $0.693^{* *}$ & 0.014 & 0.152 & -0.017 & $0.538^{* *}$ & $0.340^{* *}$ & $-0.404^{* *}$ & $0.314^{* * *}$ & -0.124 & 0.16 & $0.562^{* *}$ & $0.380^{* * *}$ \\
\hline \multirow{2}{*}{$\begin{array}{l}\text { Plant } \\
\text { height } \\
(\mathrm{cm})\end{array}$} & $\mathbf{P}$ & & & $0.850^{* *}$ & -0.077 & 0.072 & -0.13 & $-0.386^{* *}$ & 0.126 & $0.278^{* *}$ & -0.109 & 0.16 & 0.028 & $-0.312^{* *}$ & $0.335^{\text {** }}$ & $-0.207^{*}$ \\
\hline & G & & & $0.879^{* *}$ & -0.12 & 0.026 & -0.182 & $-0.432^{* *}$ & 0.15 & $0.338^{* * *}$ & -0.12 & 0.196 & 0.032 & $-0.341^{* *}$ & $0.420^{* *}$ & $-0.233^{*}$ \\
\hline \multirow{2}{*}{$\begin{array}{l}\text { Technical } \\
\text { height }(\mathbf{c m})\end{array}$} & $\mathbf{P}$ & & & & -0.168 & 0.012 & -0.083 & $-0.276^{* *}$ & 0.161 & $0.266^{*}$ & -0.136 & 0.032 & 0.102 & $-0.237^{*}$ & $0.319^{* *}$ & -0.132 \\
\hline & G & & & & -0.205 & -0.031 & -0.152 & $-0.309^{* *}$ & 0.181 & $0.327^{* *}$ & -0.159 & 0.032 & 0.12 & $-0.242^{*}$ & $0.419^{* *}$ & -0.138 \\
\hline \multirow{2}{*}{$\begin{array}{l}\text { Primary } \\
\text { branches } \\
\text { per plant }\end{array}$} & $\mathbf{P}$ & & & & & -0.005 & 0.055 & -0.021 & 0.056 & 0.065 & $-0.245^{*}$ & $0.211^{*}$ & -0.087 & 0.062 & 0.201 & 0.049 \\
\hline & G & & & & & -0.039 & 0.035 & -0.002 & 0.081 & 0.078 & $-0.253^{*}$ & $0.220^{*}$ & -0.098 & 0.071 & $0.230^{*}$ & 0.062 \\
\hline \multirow{2}{*}{$\begin{array}{l}\text { Secondary } \\
\text { branches } \\
\text { per plant }\end{array}$} & $\mathbf{P}$ & & & & & & -0.006 & -0.203 & 0.092 & 0.068 & -0.172 & 0.078 & -0.057 & 0.16 & 0.046 & 0.154 \\
\hline & G & & & & & & -0.015 & $-0.238^{*}$ & 0.123 & 0.085 & -0.173 & 0.101 & -0.059 & 0.197 & 0.055 & 0.188 \\
\hline \multirow{2}{*}{$\begin{array}{l}\text { Capsules } \\
\text { per } \\
\text { plant }\end{array}$} & $\mathbf{P}$ & & & & & & & $0.502^{* *}$ & $0.262^{*}$ & 0.158 & 0.146 & 0.078 & -0.061 & 0.067 & 0.12 & $0.239^{*}$ \\
\hline & G & & & & & & & $0.657^{* *}$ & $0.309^{* *}$ & $0.236^{*}$ & 0.162 & 0.069 & -0.084 & 0.075 & $0.213^{*}$ & $0.259^{*}$ \\
\hline \multirow{2}{*}{$\begin{array}{l}\text { Seeds per } \\
\text { capsule }\end{array}$} & $\mathbf{P}$ & & & & & & & & -0.047 & -0.122 & 0.197 & -0.05 & -0.185 & $0.219^{*}$ & -0.19 & 0.203 \\
\hline & G & & & & & & & & -0.049 & -0.151 & $0.224^{*}$ & -0.073 & -0.204 & $0.244^{*}$ & $-0.221^{*}$ & $0.239^{*}$ \\
\hline \multirow{2}{*}{$\begin{array}{l}\text { Aerial } \\
\text { biomass(g) }\end{array}$} & $\mathbf{P}$ & & & & & & & & & $0.809^{* *}$ & 0.044 & -0.019 & $0.225^{*}$ & 0.039 & $0.806^{* *}$ & $0.582^{* *}$ \\
\hline & G & & & & & & & & & $0.867^{* *}$ & 0.022 & -0.007 & $0.262^{*}$ & 0.083 & $0.851^{* * *}$ & $0.592^{* *}$ \\
\hline \multirow{2}{*}{$\begin{array}{l}\text { Straw } \\
\text { yield }(g)\end{array}$} & $\mathbf{P}$ & & & & & & & & & & -0.003 & -0.092 & $0.317^{* *}$ & $-0.266^{*}$ & $0.884^{* *}$ & $0.216^{*}$ \\
\hline & G & & & & & & & & & & -0.009 & -0.102 & $0.358^{* * *}$ & $-0.278^{* *}$ & $0.953^{* *}$ & $0.222^{*}$ \\
\hline \multirow{2}{*}{$\begin{array}{l}\text { Oil content } \\
(\%)\end{array}$} & $\mathbf{P}$ & & & & & & & & & & & -0.172 & 0.106 & 0.018 & -0.192 & 0.114 \\
\hline & G & & & & & & & & & & & -0.193 & 0.131 & 0.012 & $-0.221^{*}$ & 0.103 \\
\hline \multirow{2}{*}{$\begin{array}{l}1000 \text { seed } \\
\text { weight }\end{array}$} & $\mathbf{P}$ & & & & & & & & & & & & 0.007 & $0.279^{* *}$ & -0.006 & $0.222^{*}$ \\
\hline & G & & & & & & & & & & & & 0.008 & $0.295^{* *}$ & 0.005 & $0.240^{*}$ \\
\hline \multirow{2}{*}{$\begin{array}{l}\text { Fibre } \\
\text { yield }(g)\end{array}$} & $\mathbf{P}$ & & & & & & & & & & & & & -0.044 & $0.269^{*}$ & 0.099 \\
\hline & G & & & & & & & & & & & & & -0.048 & $0.309^{* *}$ & 0.111 \\
\hline \multirow{2}{*}{$\begin{array}{l}\text { Harvest } \\
\text { index }(\%)\end{array}$} & $\mathbf{P}$ & & & & & & & & & & & & & & $-0.297^{* * *}$ & $0.810^{* *}$ \\
\hline & G & & & & & & & & & & & & & & $-0.296^{* *}$ & $0.840^{* * *}$ \\
\hline \multirow{2}{*}{$\begin{array}{l}\text { Retted } \\
\text { straw yield } \\
\text { (g) }\end{array}$} & $\mathbf{P}$ & & & & & & & & & & & & & & & 0.185 \\
\hline & G & & & & & & & & & & & & & & & 0.183 \\
\hline
\end{tabular}


Table.2 Estimates of direct and indirect phenotypic $(\mathrm{P})$ and genotypic $(\mathrm{G})$ effects of different traits on seed yield in linseed

\begin{tabular}{|c|c|c|c|c|c|c|c|c|c|c|c|c|c|c|c|c|c|}
\hline Traits & & $\begin{array}{c}\text { Days to } \\
50 \text { per } \\
\text { cent } \\
\text { flowering }\end{array}$ & $\begin{array}{c}\text { Days } \\
\text { to } 75 \text { per } \\
\text { cent } \\
\text { maturity }\end{array}$ & $\begin{array}{c}\text { Plant } \\
\text { height } \\
(\mathrm{cm})\end{array}$ & $\begin{array}{c}\text { Technical } \\
\text { height } \\
(\mathrm{cm})\end{array}$ & $\begin{array}{c}\text { Primary } \\
\text { branches } \\
\text { per } \\
\text { plant }\end{array}$ & $\begin{array}{r}\text { Secondary } \\
\text { branches } \\
\text { per plant }\end{array}$ & $\begin{array}{c}\text { Capsules } \\
\text { per } \\
\text { plant }\end{array}$ & $\begin{array}{c}\text { Seeds } \\
\text { per } \\
\text { Capsule }\end{array}$ & $\begin{array}{c}\text { Aerial } \\
\text { biomass } \\
\text { (g) }\end{array}$ & $\begin{array}{c}\text { Straw } \\
\text { yield } \\
(\mathrm{g})\end{array}$ & $\begin{array}{c}\text { Oil } \\
\text { content } \\
(\%)\end{array}$ & $\begin{array}{c}\mathbf{1 0 0 0} \\
\text { seed } \\
\text { weight } \\
(\mathrm{g})\end{array}$ & $\begin{array}{c}\text { Fibre } \\
\text { yield } \\
(\mathrm{g})\end{array}$ & $\begin{array}{c}\text { Harvest } \\
\text { index } \\
(\%)\end{array}$ & $\begin{array}{c}\text { Retted } \\
\text { straw } \\
\text { yield }(g)\end{array}$ & $\begin{array}{c}\text { Correlatio } \\
\text { n with } \\
\text { seed yield } \\
\text { per plant }\end{array}$ \\
\hline $\begin{array}{l}\text { Days to } \\
50 \text { per cent } \\
\text { flowering }\end{array}$ & $\mathrm{P}$ & -0.0386 & 0.0528 & -0.0004 & -0.0024 & -0.0228 & 0.0026 & 0.0003 & -0.0038 & 0.1975 & -0.0132 & -0.0286 & 0.0114 & -0.0043 & 0.1481 & 0.0010 & $0.299^{* *}$ \\
\hline \multirow{2}{*}{$\begin{array}{l}\text { Days to } 75 \text { per } \\
\text { cent maturity }\end{array}$} & $\mathrm{P}$ & -0.0323 & 0.0632 & -0.0002 & -0.0022 & -0.0273 & 0.0004 & 0.0010 & -0.0013 & 0.2752 & -0.0191 & -0.0249 & 0.0097 & -0.0021 & 0.1177 & 0.0014 & $0.359^{* *}$ \\
\hline & G & -0.0095 & 0.0929 & -0.0007 & -0.0069 & -0.0637 & 0.0001 & -0.0072 & -0.0017 & 0.2627 & -0.0096 & -0.0336 & 0.0091 & -0.0035 & 0.1224 & 0.0290 & $0.380^{* *}$ \\
\hline $\begin{array}{l}\text { Plant height } \\
\text { (cm) }\end{array}$ & $\mathrm{P}$ & 0.0016 & -0.0011 & 0.0108 & -0.0283 & 0.0031 & 0.0008 & -0.0009 & -0.0169 & 0.0735 & -0.0171 & -0.0071 & 0.0053 & 0.0005 & -0.2326 & 0.0010 & $-0.207^{*}$ \\
\hline \multirow{2}{*}{$\begin{array}{l}\text { Technical } \\
\text { I height }(\mathrm{cm})\end{array}$} & $\mathrm{P}$ & -0.0028 & 0.0041 & 0.0092 & -0.0333 & 0.0069 & 0.0001 & -0.0006 & -0.0121 & 0.0940 & -0.0164 & -0.0088 & 0.0011 & 0.0020 & -0.1764 & 0.0009 & -0.132 \\
\hline & G & -0.0010 & 0.0073 & 0.0426 & -0.0878 & 0.0188 & -0.0002 & 0.0072 & -0.0308 & 0.0884 & -0.0093 & -0.0132 & 0.0009 & 0.0034 & -0.1857 & 0.0217 & -0.138 \\
\hline \multirow{2}{*}{$\begin{array}{l}\text { Primary } \\
\text { branches per } \\
\text { plant }\end{array}$} & $\mathrm{P}$ & -0.0216 & 0.0423 & -0.0008 & 0.0056 & -0.0409 & -0.0001 & 0.0004 & -0.0009 & 0.0324 & -0.0040 & -0.0159 & 0.0070 & -0.0017 & 0.0465 & 0.0006 & 0.049 \\
\hline & G & -0.0065 & 0.0644 & -0.0058 & 0.0180 & -0.0919 & -0.0003 & -0.0017 & -0.0002 & 0.0395 & -0.0022 & -0.0210 & 0.0064 & -0.0028 & 0.0541 & 0.0119 & 0.062 \\
\hline \multirow{2}{*}{$\begin{array}{l}\text { Secondary } \\
\text { branches per } \\
\text { plant }\end{array}$} & $\mathrm{P}$ & -0.0095 & 0.0023 & 0.0008 & -0.0004 & 0.0002 & 0.0105 & 0.0000 & -0.0089 & 0.0534 & -0.0042 & -0.0112 & 0.0026 & -0.0011 & 0.1194 & 0.0001 & 0.154 \\
\hline & G & -0.0031 & 0.0013 & 0.0013 & 0.0027 & 0.0036 & 0.0075 & 0.0007 & -0.0236 & 0.0599 & -0.0024 & -0.0144 & 0.0029 & -0.0017 & 0.1508 & 0.0028 & 0.188 \\
\hline $\begin{array}{l}\text { Capsules per } \\
\text { Plant }\end{array}$ & $\mathrm{P}$ & -0.0018 & 0.0092 & -0.0014 & 0.0028 & -0.0023 & -0.0001 & 0.0066 & 0.0220 & 0.1526 & -0.0097 & 0.0095 & 0.0026 & -0.0012 & 0.0502 & 0.0004 & $0.239^{*}$ \\
\hline \multirow{2}{*}{$\begin{array}{l}\text { Seeds per } \\
\text { capsule }\end{array}$} & $\mathrm{P}$ & 0.0033 & -0.0018 & -0.0042 & 0.0092 & 0.0008 & -0.0021 & 0.0033 & 0.0438 & -0.0273 & 0.0075 & 0.0128 & -0.0017 & -0.0035 & 0.1631 & -0.0006 & 0.203 \\
\hline & G & 0.0013 & -0.0016 & -0.0210 & 0.0272 & 0.0002 & -0.0018 & -0.0311 & 0.0994 & -0.0238 & 0.0043 & 0.0186 & -0.0021 & -0.0057 & 0.1867 & -0.0114 & $0.239^{*}$ \\
\hline \multirow{2}{*}{$\begin{array}{l}\text { Aerial biomass } \\
\text { (g) }\end{array}$} & $\mathrm{P}$ & -0.0131 & 0.0299 & 0.0014 & -0.0054 & -0.0023 & 0.0010 & 0.0017 & -0.0021 & 0.5825 & -0.0499 & 0.0029 & -0.0006 & 0.0043 & 0.0292 & 0.0024 & $0.582^{* *}$ \\
\hline & $\mathrm{G}$ & -0.0041 & 0.0500 & 0.0072 & -0.0159 & -0.0074 & 0.0009 & -0.0147 & -0.0048 & 0.4881 & -0.0245 & 0.0018 & -0.0002 & 0.0074 & 0.0639 & 0.0440 & $0.592^{* *}$ \\
\hline \multirow[t]{2}{*}{ Straw yield (g) } & $\mathrm{P}$ & -0.0083 & 0.0195 & 0.0030 & -0.0089 & -0.0027 & 0.0007 & 0.0010 & -0.0053 & 0.4715 & -0.0617 & -0.0002 & -0.0031 & 0.0061 & -0.1982 & 0.0026 & $0.216^{*}$ \\
\hline & G & -0.0025 & 0.0316 & 0.0164 & -0.0287 & -0.0072 & 0.0006 & -0.0112 & -0.0150 & 0.4232 & -0.0283 & -0.0008 & -0.0030 & 0.0101 & -0.2128 & 0.0493 & $0.222^{*}$ \\
\hline \multirow{2}{*}{$\begin{array}{l}\text { Oil content } \\
(\%)\end{array}$} & $\mathrm{P}$ & 0.0170 & -0.0243 & -0.0012 & 0.0045 & 0.0100 & -0.0018 & 0.0010 & 0.0086 & 0.0257 & 0.0002 & 0.0649 & -0.0057 & 0.0020 & 0.0134 & -0.0006 & 0.114 \\
\hline & G & 0.0051 & -0.0375 & -0.0058 & 0.0140 & 0.0232 & -0.0013 & -0.0077 & 0.0222 & 0.0107 & 0.0003 & 0.0832 & -0.0056 & 0.0037 & 0.0095 & -0.0114 & 0.103 \\
\hline \multirow{2}{*}{$\begin{array}{l}\text { 1000-seed } \\
\text { weight }(\mathrm{g})\end{array}$} & $\mathrm{P}$ & -0.0133 & 0.0186 & 0.0017 & -0.0011 & -0.0086 & 0.0008 & 0.0005 & -0.0022 & -0.0110 & 0.0057 & -0.0112 & 0.0332 & 0.0001 & 0.2083 & 0.0000 & $0.222^{*}$ \\
\hline & G & -0.0039 & 0.0291 & 0.0095 & -0.0028 & -0.0202 & 0.0008 & -0.0033 & -0.0073 & -0.0037 & 0.0029 & -0.0160 & 0.0289 & 0.0002 & 0.2257 & 0.0003 & $0.240^{*}$ \\
\hline \multirow{2}{*}{$\begin{array}{l}\text { Fibre yield } \\
\text { (g) }\end{array}$} & $\mathrm{P}$ & 0.0086 & -0.0070 & 0.0003 & -0.0034 & 0.0036 & -0.0006 & -0.0004 & -0.0081 & 0.1312 & -0.0196 & 0.0069 & 0.0002 & 0.0191 & -0.0331 & 0.0008 & 0.099 \\
\hline & $\mathrm{G}$ & 0.0026 & -0.0116 & 0.0015 & -0.0106 & 0.0090 & -0.0004 & 0.0040 & -0.0203 & 0.1278 & -0.0101 & 0.0109 & 0.0002 & 0.0282 & -0.0366 & 0.0160 & 0.111 \\
\hline \multirow{2}{*}{$\begin{array}{l}\text { Harvest index } \\
(\%)\end{array}$} & $\mathrm{P}$ & -0.0077 & 0.0100 & -0.0034 & 0.0079 & -0.0026 & 0.0017 & 0.0005 & 0.0096 & 0.0228 & 0.0164 & 0.0012 & 0.0093 & -0.0009 & 0.7459 & -0.0009 & $0.810^{* *}$ \\
\hline & G & -0.0024 & 0.0148 & -0.0165 & 0.0213 & -0.0065 & 0.0015 & -0.0036 & 0.0242 & 0.0407 & 0.0079 & 0.0010 & 0.0085 & -0.0014 & 0.7661 & -0.0153 & $0.840^{* *}$ \\
\hline \multirow{2}{*}{$\begin{array}{l}\text { Retted straw } \\
\text { yield (g) }\end{array}$} & $\mathrm{P}$ & -0.0128 & 0.0310 & 0.0036 & -0.0106 & -0.0082 & 0.0005 & 0.0008 & -0.0083 & 0.4696 & -0.0545 & -0.0125 & -0.0002 & 0.0051 & -0.2216 & 0.0029 & 0.185 \\
\hline & $\mathrm{G}$ & -0.0042 & 0.0522 & 0.0203 & -0.0368 & -0.0211 & 0.0004 & -0.0101 & -0.0220 & 0.4156 & -0.0269 & -0.0184 & 0.0001 & 0.0087 & -0.2271 & 0.0517 & 0.183 \\
\hline
\end{tabular}

Residual effects $(P)=0.029 ;(G)=0.006 ;$ Bold values indicates direct effects; $*$ Significant at 5 per cent level $; * *$ Significant at 1 per cent level 
The significant positive correlation of days to 50 per cent flowering with seed yield was mainly due to indirect effects via aerial biomass, harvest index and days to maturity, whereas indirect effects via other traits were low in magnitude.

The significant positive correlation of days to 75 per cent maturity with seed yield was mainly due to positive direct effect on seed yield and also indirect effect via harvest index and aerial biomass, whereas it exhibited low magnitude of negative indirect effects via straw yield, oil content and primary branches per plant.

Positive correlation of capsules per plant was mainly due to aerial biomass and harvest index, whereas low magnitude of indirect effects via days to 75 per cent maturity and capsules per plant.

Aerial biomass exhibited high positive direct effect with seed yield per plant and low negative indirect effect via days to 50 per cent flowering, seeds per capsule and straw yield. 1000 -seed weight high indirect effects via harvest index followed by 75 per cent maturity and negative indirect effects via aerial biomass.

For harvest index, positive indirect effects were mainly exhibited via., days to 75 per cent maturity, secondary branches per plant, straw yield, oil content and aerial biomass.

Similar results were observed by Tadesse et al., (2009) for harvest index and aerial biomass. Bindra (2012), Patial (2014) and Bindra and Paul (2016) revealed that aerial biomass was the main determinant of seed yield per plant. Similar studies on path coefficient analysis were done by Chandra (1978), Muduli and Patnaik (1994) and Chimurkar et al., (2001). They observed direct effect of capsules per plant and 1000- seed weight on seed yield per plant. Akbar et al., (2003) in a similar study on path coefficient analysis revealed that the number of capsules per plant had highest direct effect followed by plant height, 1000-seed weight and number of branches per plant on seed yield per plan, whereas results of present findings revealed that harvest index and aerial biomass contributed directly towards seed yield. Thus, revealing the importance of aerial biomass rather than number of capsules per plant as suggested by previous workers (Chandra 1978; Muduli and Patnaik 1994; Chimurkar et al., 2001; Muhammad et al., 2003, Reddy et al., 2013, Rajanna et al., 2014 and Pali and Mehta 2016) for increasing the seed yield per plant through direct selection.

\section{References}

Akbar, M. Mahmood, T. Anwar, M. Ali, M. Shafiq, M. and Salim, J. 2003. Linseed improvement through genetic variability, correlation and path coefficient analysis. International Journal Agriculture Biology, 5: 303305.

Al-Jibouri, H. A. Millar, P. A. and Robinson, H. P. 1958. Genotypic and environmental variance and covariances in an upland cotton cross of interspecific origin. Agronomy Journal, 50: 633-637.

Badwal, S.S. Gill, K.S. and Singh, H. 1970. Path coefficient analysis of seed yield in linseed. Indian Journal of Genetics and Plant Breeding, 30: 551-556.

Belete, Y.S. and Yohannes, M.T.W. 2013. Genetic variation of different crosses of linseed genotypes for some Agromorphological Traits. Asian Journal of Crop Science, 5: 436-443.

Bibi, T. Mahmood, T. Mirza, T. Mahmood, T. and Ejaz-ul-Hasan. 2013. Correlation studies of some yield related traits in linseed (Linum usitatissimum L.). 
Journal of Agricultural Sciences, 51: 121-132.

Bindra, S. 2012. Genetic diversity and association studies in linseed (Linum usitatissimum L.). M.Sc thesis, Department of crop improvement, CSK Himachal Pradesh Krishi Vishvavidyalaya, Palampur, India.

Bindra, S. and Paul, S. 2017. Genetic Variability and Association Studies in linseed (Linum usitatissimum L.). An International Quarterly Journal of Life Sciences, 11: 1855-1859.

Chandra, S. 1978. Studies of interrelationship between seed yield and its components in some exotic strains of linseed (Linum usitatissimum L.). Acta Agronomica Hungarica, 27: 74-80.

Chimurkar, H.C. Shanti, P. and Rathod, D.R. 2001. Character association studies in linseed (Linum usitatissimum L.). Annals of Plant Physiology, 15: 72-76.

Copur, O. and Oglakci, M. 1998. A research on thec orrelation and path coefficient analysis of yield and yield components of cotton (Gossypium hirsutum L.). In Harran Plain Conditions. Harran University. Journal of Faculty of Agriculture Kyushu University, 2: 1120.

Dewy, D.R. and Lu, K.H. 1959. A correlation and path coefficient analysis of components of crested wheat grass seed production. Agronomy Journal, 51: 515-518.

Gauraha, D. and Rao, S.S. 2011. Association Analysis for Yield and its Characters in Linseed (Linum usitatissimum L.). Research Journal of Agricultural Sciences, 2: 258-260.

Gill, K.S. 1987. Linseed. ICAR publication, New Delhi, India.

Gul, S. Rajper, A.A. Kalhoro, F.A. Kalhoro, S.A. Ali, A. Shah, F.A. Raza, S. Ahmed, M. 2016. Screening Selected Linseed (Linum usitatissimum L.)
Genotypes for Yield Performance in Sindh, Pakistan. Natural Science. 8: 5365.

Gupta, T.R. Pal, S.S. and Singh, I. 1999. Parameters of genetic variability and correlation studies in linseed (Linum usitatissimum L.). Journal Oilseeds Research, 16: 213-215.

Joshi, B.K. Mudwari, A. Bhatta, M.R. Ferrara, G.O. 2004. Genetic diversity in Nepalese wheat cultivars based on agromorphological traits and coefficients of parentage. Nepal Agriculture Research Journal, 5:7-17.

Kant, R. Chauhan, M.P. Srivastava, R.K. and Maurya, K.N. 2008. Correlation and path coefficient analysis of seed yield and yield components of linseed (Linum usitatissimum L.). International Journal of Plant Science, 3: 323-325.

Khan, M.L., Sharif, M. and Sarwa, M. 2010. Chemical Composition of Different Varieties of Linseed. Pakistan Veterinary Journal, 2: 33-38.

Kumar, N and Paul, S. 2016. Selection criteria of linseed genotypes for seed yield traits through correlation, path coefficient and principal component analysis. The Journal of Animal \& Plant Sciences, 26: 1688-1695.

Mahto, C. and Rahaman, M.H. 1998. Correlation and path analysis of some quantitative characters in linseed. Journal of Oilseeds Research, 15: 348351.

Muduli, K.C. and Patnaik, M.C. 1994. Character association and path coefficient analysis in linseed (Linum usitatissimum L.). Orissa Journal of Agricultural Research, 7: 6-11.

Muhammad, A. Mahmood, T. Anwar, M. Muhammad, A. Muhammad, S. and Jafar, S. 2003. Linseed improvement through genetic variability, correlation and path coefficient analysis. International Journal of Agricultural 
and Biology, 5: 303-305.

Naik, B.S. and Satapathy, P.C. 2002. Selection strategy for improvement of seed yield in late sown linseed. The Journal of Agriculture and Crop Research, 3: 599-605.

Pali, V. and Mehta, N. 2015. Character association analysis for seeds Yield and its components in linseed (Linum usitatissimum L.). Trends in Biosciences, 8: 4573-4576.

Patial, R. 2014. Genetic characterization of elite genotypes of linseed (Linum usitatissimum L.) M.Sc Thesis. Department of Plant Breeding and Genetics, CSK Himachal Pradesh Krishi Vishvavidyalaya, Palampur, India.

Paul, S. Bhateria, S. and Kumari, A. 2015. Genetic variability and interrelationships of seed yield and yield components in linseed (Linum usitatissimum L.). Journal Breeding Genetics, 47: 375-383.

Paul, S. Kumar, N. and Chopra, P. 2017. Correlation and genetic diversity of linseed (Linum usitatissimum L.) genotypes based on principal component analysis in Mid-Hills of North-West Himalayas. Journal of Pharmacognosy and Phytochemistry, 6: 287-290.

Rajanna, B. Biradar, S.A. and Ajithkumar, K. 2014. Correlation and path coefficient analysis for yield and yield components in linseed (Linum usitatissimum L.) An
International Quarterly Journal of Life Science, Supplement on Genetics and Plant Breeding, 9: 1625-16.

Reddy,M.P. Reddy, R.B. Arsul, B.T. and Maheshwari, J.J. 2013. Character association and Path Coefficient Studies in Linseed. International Journal of Current Microbiology and Applied Science, 2: 250-254.

Sarkar, T.K. 2005. Genetic variability and character association in linseed (Linum usitatissimum L.). M.Sc. Thesis, Orissa University of Agriculture \& Technology, Bhubaneswar, Odisha, India.

Sohan, R. Singh, S.K. and Kerketta, V. 2004. Correlation studies in linseed (Linum usitatissimum L.). Birsa Agriculture University Journal of Research, 16: 123- 126.

Tadesse, T. Singh, H. and Weyessa, B. 2009. Correlation and path coefficient analysis among seed yield traits and oil content in Ethiopian linseed Germplasm. International Journal of Sustainable Crop Production, 4: 08-16.

Wright, S. 1960. Theory of path coefficient and regression; alternative or complementary concepts. Biometrics, 16: $189-202$.

Yucel, C. 2004. Correlation and path coefficient analysis of seed yield components in the narbon bean (Vicia narbonensis L.). Turkish Journal of Agriculture and Forestry, 28: 371-376.

\section{How to cite this article:}

Navdeep Kaur and Satish Paul. 2020. Correlation and Path Coefficient Analysis for Seed Yield and Fibre Traits in Linseed (Linum usitatissimum L.). Int.J.Curr.Microbiol.App.Sci. 9(02): 6675. doi: https://doi.org/10.20546/ijcmas.2020.902.009 